\title{
A New Sensor Signal Processing Technology of Multi-phase PMSM Rotor Position Detection
}

\author{
Shuang $\mathrm{YU}^{1, \mathrm{a}}$, Yan $\mathrm{LI}^{1}$, Jiakuan $X \mathrm{IA}^{1}$, Haochi JIANG ${ }^{1}$ \\ ${ }^{1}$ Research Institute of Electric Drive, Shenyang University of Technology, Shenyang, 110870, China \\ a email: yushuang1124@126.com
} Keywords: Multi-phase PMSM; Rotor Position Detection; Incremental Photoelectric Encoder;
E²PROM

\begin{abstract}
This paper proposes a new sensor signal processing technology to detect rotor position in permanent magnet synchronous motor (PMSM). This technology uses an incremental photoelectric encoder and some hardware circuit. It is easy to achieve, and it is not only apply to the traditional three-phase PMSM, but also suitable for the multi-phase symmetry or asymmetry PMSM. The calculation and theoretical analysis show that, this new sensor signal processing technology has high detection accuracy. The mentioned technology can meet the requirement of actual use when using in the experimental system.
\end{abstract}

\section{Introduction}

With the advantages of simple structure, high efficiency, low noise, reliable operation, wide speed range and running at very low frequencies, PMSM is widely used in AC servo system. Accurate detection of the rotor position information is the foundation of PMSM vector control [1]. Inaccurate rotor position detection can not achieve high accuracy, high dynamic response of the motor position and speed control. More seriously, it will cause the motor does not work properly, for these reasons, the position of the rotor pole permanent magnet synchronous motor precise positioning is particularly important.

Multiphase PMSM is developed on the basis of three-phase PMSM, it is applied in many fields, such as aerospace, aviation, marine electric propulsion. Compared with ordinary three-phase PMSM, Multiphase PMSM have many advantages. With the increase of number of phases, the number of current harmonics minimum times increases and the amplitude of current harmonic decreases, which improve system stability and reduce torque ripple and improve motor efficiency, while it can reduce the rotor harmonic losses[2]. Structurally, it comprises two forms, namely symmetrical and asymmetrical. For asymmetric multiphase PMSM, its rotor is asymmetry in the magnetic and electrical structural, so that it is difficult to determine the rotor pole position using the general control algorithm. This paper presents the method of rotor position detection which not only applies to traditional symmetrical multiphase PMSM, but also applies to asymmetric multiphase PMSM.

\section{Common Methods of Rotor Position Detection}

At present, the common method of PMSM rotor position detection is generally divided into two types: detection without position sensor or with position sensor.

Detection without Position Sensor. The method of detecting rotor position without sensor is a keen research direction among scholars, which is through the analysis and calculation of motor parameters, estimate the approximate position of the rotor, such as counter electromotive force method [3], high-frequency signal injection method [4], fretting rotor method [5]. These methods are excessive dependence motor parameters obviously there are many drawbacks.

Detection with Position Sensor. Compared with detection without position sensor, detection rotor position by the position sensor is the most direct and effective method. Currently, there are three commonly used position sensors: Hall sensor [6], Rotary encoder [7] and Optical encoder [8]. 
Hall sensor is small, easy installation, low price and does not require mechanical coupling device. Switch hall, which generates discrete position signal, is widely used in permanent magnet brushless DC (BLDC) motor, so that it generally can not be directly used for PMSM vector control system. However, the linear Hall can provide continuous position information, but its output tends to contain a lot of harmonic, seriously affect the accuracy of the location estimation.

Rotary transformer is one of detecting devices, which is commonly used to detect rotor position. Its advantage is high reliability and strong anti-jamming capability, but the rotary transformer demodulation is required, and the signal processing circuit is more complex.

Optical encoder, divided into two kinds of absolute and incremental, is the most widely used method to detect the rotor position currently. The absolute encoder can directly output the absolute position of the rotor, do not need determine the rotor initial position, but the price is more expensive. The incremental photoelectric encoder with the positioning signal of $\mathrm{U}, \mathrm{V}, \mathrm{W}$, can be used to detect the initial position of the PMSM rotor, besides it has the advantages of small size, fast response, and low price.

\section{Signal Processing Technology of the Incremental Photoelectric Encoder}

Incremental Photoelectric Encoder. As is shown in Figure 1, the incremental photoelectric encoder will produce three pulse signals: "A", "B", and "Z", during the code disc rotation.

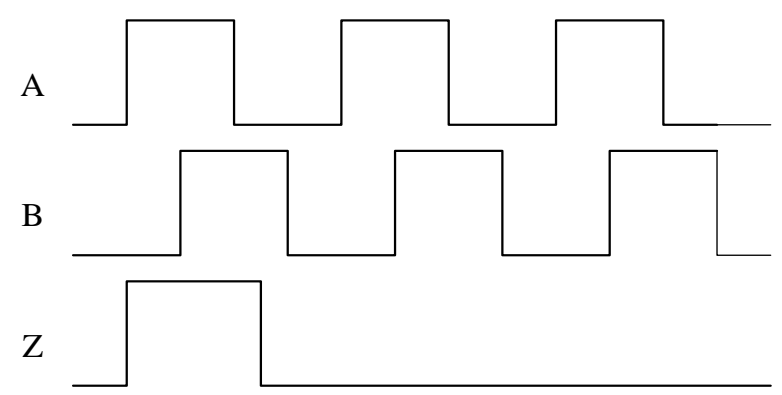

Fig.1. The output wave of Incremental encoder

The phase relationship between the pulse signal "A" and "B" are orthogonal, and they have the same frequency, so the motor is rotated forward or reverse rotation can be known by determining the phase relationship between pulses signal "A" and "B". The encoder sends only one "Z" pulse in a period of rotation, the signal " $Z$ " can be used to eliminate the Pulses interference or loss of pulses which will cause the position counter to produce cumulative error.

The incremental photoelectric encoder with the positioning signal of "U", "V", "W", can be used to detect the initial position of the PMSM rotor, as is shown in Figure 2, the three pulse signal "U", "V", "W" between the difference of 120 electrical degrees in an electric angle cycle, the output of these three signals composed of 6 states, 60 electrical degrees each state. In order to carry out the initial position of motor rotor, the rising edge of the "Z" encoder signal "U" should be leveled with the rising edge of the motor "A" phase's back-EMF zero crossing point from negative to positive in the beginning, at this point, the axis of motor winding " $\mathrm{A}$ " is coincidence with the $\mathrm{D}$ axis of the rotor.

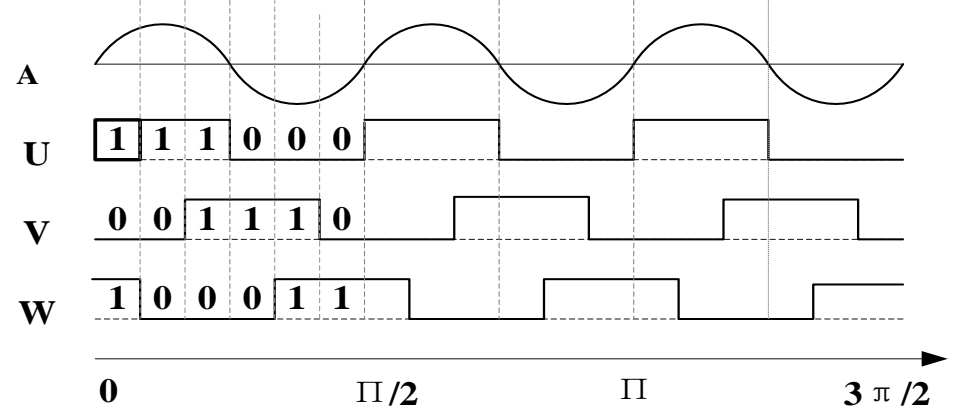

Fig.2 The back-EMF of motor "A" phase and the output "UVW" of encoder 
When the encoder is installed, the position of the encoder signal " $\mathrm{U}$ " and " $\mathrm{Z}$ " is fixed, and they have corresponding relations between the axis of "A" phase, but the motor rotor position is random. When the system is powered, the interval of the rotor (0-5) can be determined by detecting the three-phase state of the "U", "V", "W". Because of the U, V, W can distinguish the 60 electrical degrees, with section " 0 " as an example, the electric angle range between 0 to 60 degrees, so use the median value 30 degrees to represent the current position. After determining the initial position of rotor, the motor starts rotating, the position of the rotor will be revised until the arrival of the $\mathrm{Z}$ pulse signal, then use pulse signal "A" and "B" to obtain accurate rotor position signal.

Techniques of Sensor Signal Processing. The motor winding phase "A" axis as the dividing line, the encoder rotation motor can be divided into $\mathrm{N}$ regions, i.e.,

$$
N=\frac{360^{\circ} \mathrm{p}}{\theta}
$$

Where $\mathrm{p}$ is the number of pole-pairs and $\theta$ is the minimum angle between each phase of the Multi-phase motor.

In order to facilitate the description, from the beginning of the phase " $\mathrm{A}$ " winding axis, along the rotation direction of the rotor, the region is denoted as $1 \sim \mathrm{N}$, that is to say, the motor will occur $\mathrm{N}$ times' commutation in a circle. With the encoder rotates with the motor rotor axial, each region contains $n$ times' pulse, each n times' pulse will control the motor to commutate one time.

The number of the encoder pulse in each region of the Multi-phase motor

$$
\mathrm{n}=\frac{4 r}{N}
$$

Where $4 \mathrm{r}$ is the number of pluses in a rotation cycle, after quadrupling the resolution of $\mathrm{R}$ encoder.

An encoder generates pulses can be obtained by counting, counter starts counting from 0 until the $\mathrm{Z}$ pulse arrival after the motor started. Corresponding to the information of rotor position and commutation information to stored in memory, the memory address line inputs for the rotor position information, while the data line output the motor commutation information. The hardware specific flow chart is shown in Figure 3.

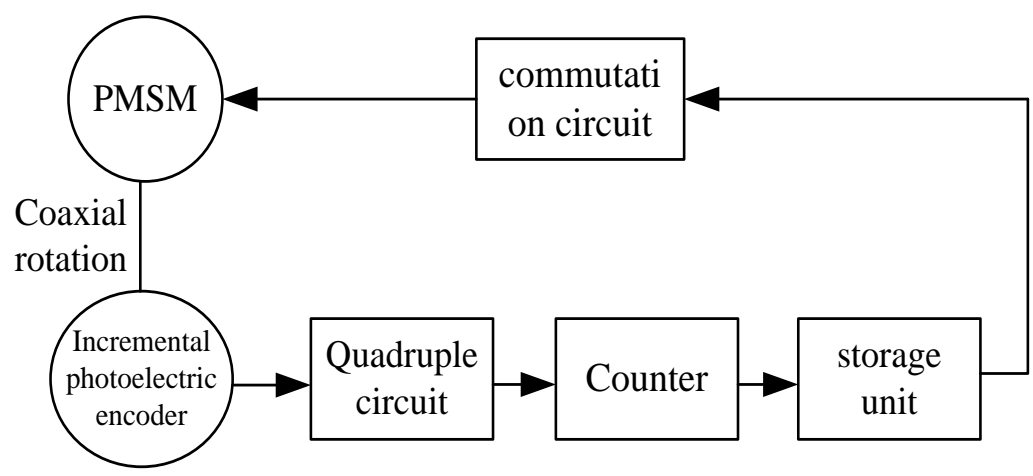

Fig.3 The hardware flow chart

Use the electrically erasable programmable read-only memory ( $\left.E^{2} \mathrm{PROM}\right)$ with parallel port I/O as the storage unit. The number of $E^{2} \mathrm{PROM}$ address line is

$$
A=\left[\log 2^{4 r}\right]+1
$$

The number of $\mathrm{E}^{2} \mathrm{PROM}$ data lines is determined according to the number of motor phases. The capacity of the memory can be determined by the following formula:

$$
R=\frac{2^{A}}{2^{10}} K \times \mathrm{m}
$$

Where $\mathrm{m}$ is the number of motor phases, and $\mathrm{K}$ is the unit of capacity . 


\section{Example}

Take the four-pole asymmetrical six-phase PMSM (also called "double three-phase PMSM") as an example. Figure 4 is the structure diagram of double three-phase PMSM which can observe the minimum angle between each phase is 30 degree.

By using the method of detecting the rotor position above, as Eq.1 shows, the motor can be divided into 48 areas with the encoder rotation. As shown in Eq.2, after quadrupling, 2500PPR encoder, each region contains about 208 pulses. In this example, the asymmetric six-phase motor will produce 12 commutation states in an electric angle cycle. As shown in Figure 5, it starts from the axis of the motor phase windings "A", they are $100011 \rightarrow 110011 \rightarrow 110001 \rightarrow 110000 \rightarrow 111000 \rightarrow 111100$ $\rightarrow 011100 \rightarrow 001100 \rightarrow 001110 \rightarrow 001111 \rightarrow 000111 \rightarrow 000011$.

According to the calculation, the corresponding relationship between the encoder pulse and the information about the commutation can be obtained, then write the data table of commutation information to stored to E ${ }^{2}$ PROM in advance for addressing the call. By the Eq.3 and Eq.4 can be calculated, the $\mathrm{E}^{2} \mathrm{PROM}$ need 14 bits address lines and 12 bits data lines, the memory capacity is $64 \mathrm{~K}$ $\times 12$. Table 1 lists the correspondence between $E^{2} P R O M$ 's input-output and the rotor position in a pair of pole.

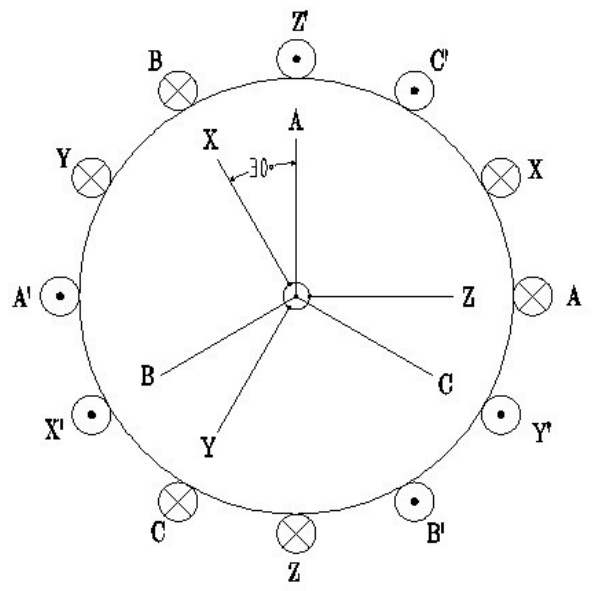

Fig.4 The structure diagram of asymmetrical six-phase PMSM

\begin{tabular}{|c|c|c|c|}
\hline $\begin{array}{c}\text { Rotor } \\
\text { Position } \\
\end{array}$ & pulses & E2PROM Address & $\begin{array}{c}E^{2} \text { PROM } \\
\text { Output } \\
\end{array}$ \\
\hline Zone 1 & 208 & 00000011010000 & 100011 \\
\hline Zone 2 & 417 & 00000110100001 & 110011 \\
\hline Zone 3 & 625 & 00001001110001 & 110001 \\
\hline Zone 4 & 833 & 00001101000001 & 110000 \\
\hline Zone 5 & 1042 & 00010000010010 & 111000 \\
\hline Zone 6 & 1250 & 00010011100010 & 111100 \\
\hline Zone 7 & 1458 & 00010110110010 & 011100 \\
\hline Zone 8 & 1667 & 00011010000011 & 001100 \\
\hline Zone 9 & 1875 & 00011101010011 & 001110 \\
\hline Zone 10 & 2083 & 00100000100011 & 001111 \\
\hline Zone 11 & 2292 & 00100011110100 & 000111 \\
\hline Zone 12 & 2500 & 00100111000100 & 000011 \\
\hline$\cdots$ & $\cdots$ & $\cdots$ & $\cdots$ \\
\hline
\end{tabular}

Fig.5 The phase relationship of asymmetrical six-phase PMSM.
Tab.1. The data table of $\mathrm{E}^{2} \mathrm{PROM}$

\begin{tabular}{c|c|c|c}
\hline $\begin{array}{c}\text { Rotor } \\
\text { Position }\end{array}$ & pulses & E2PROM Address & $\begin{array}{c}\text { E2PROM } \\
\text { Output }\end{array}$ \\
\hline Zone 1 & 208 & 00000011010000 & 100011 \\
Zone 2 & 417 & 00000110100001 & 110011 \\
Zone 3 & 625 & 00001001110001 & 110001 \\
Zone 4 & 833 & 00001101000001 & 110000 \\
Zone 5 & 1042 & 00010000010010 & 111000 \\
Zone 6 & 1250 & 00010011100010 & 111100 \\
Zone 7 & 1458 & 00010110110010 & 011100 \\
Zone 8 & 1667 & 00011010000011 & 001100 \\
Zone 9 & 1875 & 00011101010011 & 001110 \\
Zone 10 & 2083 & 00100000100011 & 001111 \\
Zone 11 & 2292 & 00100011110100 & 000111 \\
Zone 12 & 2500 & 00100111000100 & 000011 \\
\hline
\end{tabular}

The concrete realization process is as follows:

Firstly, determine the initial position of rotor by the signals "U", "V", "W" of the incremental encoder, so that the motor can begin to rotate. Until the encoder pulse signal " $Z$ " arrives, the 
counter starts to count from zero. Secondly, the corresponding binary codes are input to memory address line when the counter counts to 208 (or 209) pulses, then determine the location of the rotor position as "Zone 1" by the memory addressing. Finally, call the commutation information of the memory, and output of the first binary code 100011 to bridge inverter circuit to control power switches on or off, so that realize the commutation of the PMSM. With the encoder and the motor continues to rotate, the counter continues to count pulses, when the count reaches 416, the rotor position as "Zone 2", the output commutation information becomes 110011 . And so on, we can obtain accurate position signal of rotor space, providing the right commutation information for the motor.

\section{Conclusion}

In this paper, a new sensor signal processing technology is proposed to detect rotor position. With this processing, even if the motor control chip crashes, the system still can detect sensor signal processing, the motor remains relatively stable operation, and will not appear sensor signal errors or be lost. Practice has proved that this method is suitable for multi-phase PMSM, and positioning accuracy is high. It has great significance in the practical application of AC servo system.

\section{Acknowledgement}

In this paper, the research was sponsored by Program for LNIRT in university (LT2013006).

\section{References}

[1] Application of neural networks for permanent magnet synchronous motor direct torque control [J]. Journal of Systems Engineering and Electronics, 2008, 03:555-561.

[2] Ouyang H, Zhou M, Tong T. Vector Control of Multi-Phase Permanent Magnet Synchronous Motor with Asymmetric Structure[J]. PROCEEDINGS-CHINESE SOCIETY OF ELECTRICAL ENGINEERING, 2004, 24(7): 145-150.

[3] Ertugrul, Nesimi, and Paul Acarnley. A new algorithm for sensorless operation of permanent magnet motors [J]. Industry Applications, IEEE Transactions on 30.1 (1994): 126-133.

[4] Jang, Ji-Hoon, et al. Sensorless drive of surface-mounted permanent-magnet motor by high-frequency signal injection based on magnetic saliency [J]. Industry Applications, IEEE Transactions on 39.4 (2003): 1031-1039.

[5] Yituo LI, et al. A novel initial rotor position estimation method for permanent magnet synchronous motors [J]. Proceedings of the Chinese Society of Electrical Engineering. Vol. 33. No. 3. Chinese Society for Electrical Engineering, 2013.

[6] XU Yong-xiang, HU Jian-hui, ZOU Ji-bin, et al. Design of the Rotor Position Detecting Circuit Based on One Discrete Hall Sensor [J]. Chinese Journal of Electron Devices 2 (2008):465-468.

[7] Cui Jun, Wen Xuhui, Zhang Liwei. Application of a Novel Resolver-digital Converter in PMSM Control System [J]. Electric Drive 11 (2005): 002.

[8] Rong, CHEN. Rotor Incipient Position Location of PMSM Based on Incremental Photoelectric Coded Disk [J]. Electric Machines \& Control Application 3 (2007): 008. 